\title{
Adequate Dextran Sodium Sulfate-induced Colitis Model in Mice and Effective Outcome Measurement Method
}

\author{
Yo Han Park', Nayoung Kim ${ }^{1,2}$, Young Kwang Shim¹, Yoon Jin Choi', Ryoung Hee Nam', Yoon Jeong Choi ${ }^{1}$, \\ Min Hee Ham ${ }^{1}$, Ji Hyung Suh ${ }^{1}$, Sun Min Lee', Chang Min Lee ${ }^{1}$, Hyuk Yoon ${ }^{1}$, Hye Seung Lee ${ }^{3}$, Dong Ho Lee \\ Departments of ${ }^{1}$ Internal Medicine and ${ }^{3}$ Pathology, Seoul National University Bundang Hospital, Seongnam, ${ }^{2}$ Department of Internal Medicine \\ and Liver Research Institute, Seoul National University College of Medicine, Seoul, Korea
}

\begin{abstract}
Background: Dextran sodium sulfate (DSS)-induced colitis mouse model is used for research of inflammatory bowel disease. The aim of this study was to establish the adequate conditions for DSS mice model, and to find useful tool to measure inflammation. Methods: The 2.5\% DSS was administered to six male C57BL/6 mice and 4\% DSS to eight mice at 5 or 9 weeks of age. Each group was consisted of 6 mice with control group in which vehicle was administered instead of DSS. The mice were sacrificed on the 7th day after DSS or vehicle administration. Body weight, diarrhea, and hematochezia were recorded daily. Disease activity index (DAI) score which was composed of body weight change, diarrhea, and hematochezia was measured every day. Colon length was measured after sacrifice and colon mucosal level of interleukin 1 beta (IL-1 $\beta$ ) was measured by ELISA assay. Histological score was compared between ascending and descending colon in the DSS group.

Results: Colon length of five- and nine-week DSS group was significantly shorter than each control group but there was no statistical significance depending on DSS concentration or age. DAI score of 4\% DSS group in nine-week was significantly higher than that five-week $(P=0.012)$ but there was no difference between $2.5 \%$ and $4 \%$ DSS group. The level of IL-1 $\beta$ in DSS mice was much higher than control group $(P<0.01)$, but there was no difference among several DSS groups. The histological score was higher in the descending colon than in the ascending colon but there was no statistical difference between each pair of DSS groups.

Conclusions: The 4\% DSS mice in nine-week was adequate for DSS-induced colitis model. DAI score was useful tool and descending colon was more appropriate site for histological evaluation of colitis than ascending colon.

(J Cancer Prev 2015;20:260-267)
\end{abstract}

Key Words: Dextran sulfate, Mice, Colitis, Inflammatory bowel disease

\section{INTRODUCTION}

Inflammatory bowel disease (IBD) mainly comprised of ulcerative colitis (UC) and Crohn's disease (CD) is characterized by chronic inflammation of the gastrointestinal tract with multifactorial ethiology. ${ }^{1}$ When a patient presents with symptoms such as diarrhea, abdominal pain, hematochezia, and weight loss, ${ }^{2}$ then they suggestive UC or CD. For research of IBD, there are several kinds of animal models of colonic inflammation. ${ }^{3}$ Generally, IBD colitis models are classified into five groups: chemically induced model, spontaneous model, cell-transfer model, congenital model, and genetically engineered model. ${ }^{3}$ Among various mice colitis models, dextran sodium sulfate (DSS)-induced colitis is widely used because of its simplicity, time- and cost-saving. ${ }^{3.4}$ First report of DSS-induced colitis model was published in the year 1985 , by Ohkusa, ${ }^{5}$ which has some advantages in comparison with other animal models of colitis. For example, administration of DSS in drinking water provokes acute or chronic colitis depending on the administration concentration. ${ }^{6}$ Moreover, severe DSS-induced colitis closely resembles the

Received November 23, 2015, Revised December 11, 2015, Accepted December 12, 2015

Correspondence to: Nayoung Kim

Department of Internal Medicine, Seoul National University Bundang Hospital, 82 Gumi-ro 173beon-gil, Bundang-gu, Seongnam 13620, Korea Tel: +82-31-787-7008, Fax: +82-31-787-4051, E-mail: nayoungkim49@empas.com, ORCID: Nayoung Kim, http://orcid.org/0000-0002-9397-0406

Copyright (C) 2015 Korean Society of Cancer Prevention

(c) This is an Open Access article distributed under the terms of the Creative Commons Attribution Non-Commercial License (http://creativecommons.org/icenses/by-nc/4.0) which permits unrestricted non-commercial use, distribution, and reproduction in any medium, provided the original work is properly cited. 
clinical features of human UC. ${ }^{7}$ However, as there are several DSS-induced mice model protocols depending on concentration of DSS or ages of mice, ${ }^{89}$ the beginners experience difficulty in the choosing the effective condition. From this background the aim of this research was to investigate the proper model for acute IBD study by combinations of DSS concentration and ages in C57BL/6 mice. In addition, we tried to find the effective measurement method of IBD induction among colon length, body weight, diarrhea, hematochezia, histological finding, and interleukin 1 beta (IL-1 $\beta$ ).

\section{MATERIALS AND METHODS}

\section{Animals and dextran sodium sulfate}

Five or nine-week-old C57BL/6 male mice (Orient Co. Ltd., Seoul, Korea) were used for the experiments. All mice were housed in a cage maintained at $23^{\circ} \mathrm{C}$ with a $12 / 12$-hour light/dark cycle under specific pathogen-free conditions. Control and 2.5\% DSS group was administered to six male C57BL/6 mice and 4\% DSS was administered to eight mice. At first day, both control group and DSS group were administered orogastrically with vehicle only. And DSS (MP Biomedicals, Santa Ana, CA, USA; M.W 36-50 $\mathrm{kDa}$, Ref $=160110$ ) group were administered with DSS vehicle from 2 to 7 days. Both control and DSS group were sacrificed by $\mathrm{CO} 2$ asphyxiation at 7 days. The time schedule of this study is represented in Figure 1. From the day of experiment administration, all mice were labeled and the weights of mice were measured daily. Features of stool, such as diarrhea or bloody stool, from each mouse were observed every day. All experimental procedures were approved by the Institutional Animal Care and Use Committee (IACUC) of Seoul National University Bundang Hospital (IACUC number: BA1506-178-039-01).

\section{Body weight, diarrhea, hematochezia and colon lengths}

Severity and inflammation of colitis were assessed by examining body weight, diarrhea, hematochezia and colon length. The body weights of all mice were measured from the date of administration to sacrifice daily. All mice were observed for stool consistency and rectal bleeding every day. To assess the severity of colitis, disease activity index (DAI) score were monitored daily. ${ }^{10}$ The colon length was measured at sacrifice time which was performed on Day 7 of experiment.

\section{Measurement of interleukin 1 beta}

Increased pro-inflammatory cytokine is a hallmark of DSSinduced colitis. Therefore, the level of IL-1 $\beta$ in the colon was measured by ELISA assay. Ten milligrams of colon strip (one from the ascending colon $[\mathrm{AC}]$ and one from the descending colon [DC]) was homogenized for 30 seconds with a polytron homogenizer in $200 \mu \mathrm{L}$ of ice-cold lysis buffer $(200 \mathrm{mM} \mathrm{NaCl}, 5 \mathrm{mM}$ EDTA, 10 mM Tris [pH 7.4], 10 \% glycerin, 1 mM phenylmethylsulfonyl fluoride, $1 \mu \mathrm{g} / \mathrm{mL}$ leupeptin and $28 \mu \mathrm{g} / \mathrm{mL}$ aprotinin). Suspensions were centrifuged at 13,000 rpm for 15 minutes and the resulting supernatant was assayed using an IL-1 ELISA kit (R\&D Systems, Minneapolis, MN, USA) according to the manufacturer's instruction. Protein concentration was measured by using Bio-Rad protein assay kit (Bio-Rad laboratories, Hercules, MA,USA).

\section{Histopathological analysis}

Proximal and distal colonic sections were fixed in $10 \%$ formalin and embedded in paraffin. The 5-mm sections were stained with haematoxylin and eosin (H\&E). The microscopic colonic epithelial damage was assigned scores as follows: $0=$ normal; $1=$ hyperproliferation, irregular crypts, and goblet cell loss; 2 = mild to moderate crypt loss (10\%-50\%); 3 = severe crypt

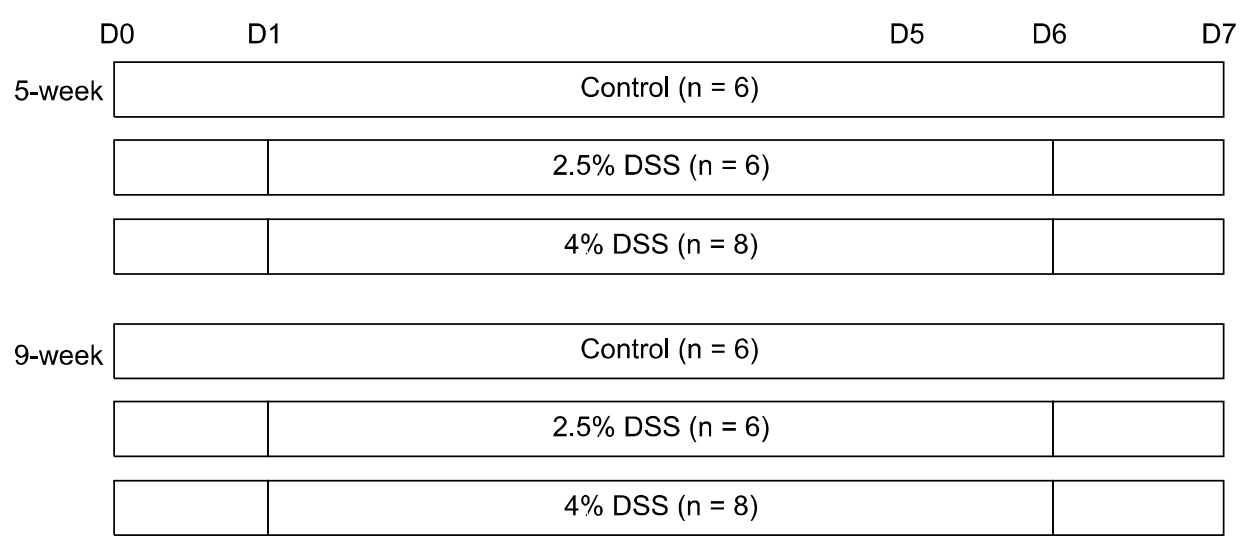

Figure 1. Schematic diagram of study design. D, day of administration; DSS, dextran sodium sulfate. 
loss $(50 \%-90 \%) ; 4=$ complete crypt loss, surface epithelium intact; $5=$ small- to medium-sized ulcer ( $<10$ crypt widths); and 6 = large ulcer ( $\geq 10$ crypt widths). Infiltration with inflammatory cells was assigned scores separately for mucosa $(0$ $=$ normal, $1=$ mild, $2=$ modest, $3=$ severe $)$, submucosa $(0=$ normal, $1=$ mild to modest, $2=$ severe $)$, and muscle/serosa $(0=$ normal, $1=$ moderate to severe). Scores for epithelial damage and inflammatory cell infiltration depending on colonic depth were added, resulting in a total scoring range of 0 to $12 .{ }^{11}$

\section{Statistical analysis}

Data are expressed as mean \pm SEM. Comparison of between the 3 groups (2.5\% DSS, 4\% DSS and control) was performed using the Kruskal-Wallis test. Comparison of between the 2 different groups was performed using the Mann-Whitney test. And Fisher's exact test was used for categorical data. $P$-values less than 0.05 were considered statistically significant. All statistical analyses were performed using IBM SPSS software ver. 22.0 (IBM Co., Armonk, NY, USA).

\section{RESULTS}

\section{Body weight, diarrhea and hematochezia}

The body weights of all mice of control and DSS group was measured daily. The change of body weight from D0 to D7 express is shown at Figure 2A. The baseline of the weight change was the
A
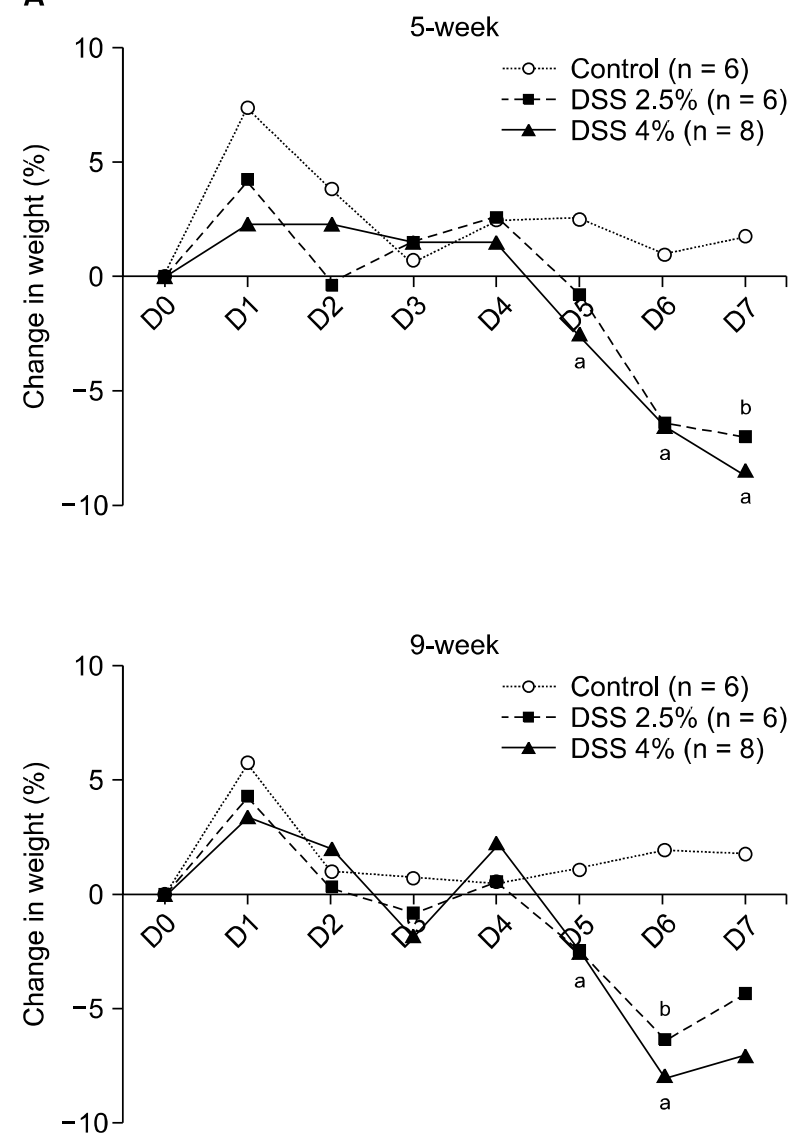

B

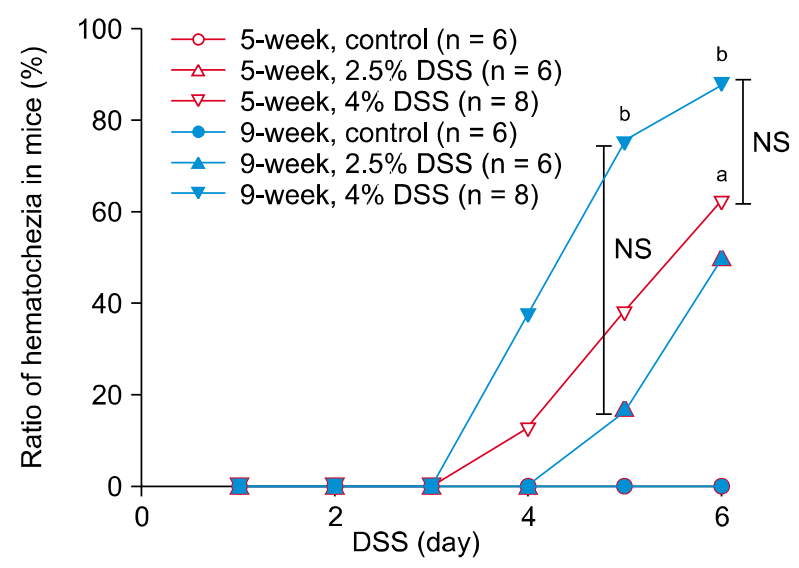

C

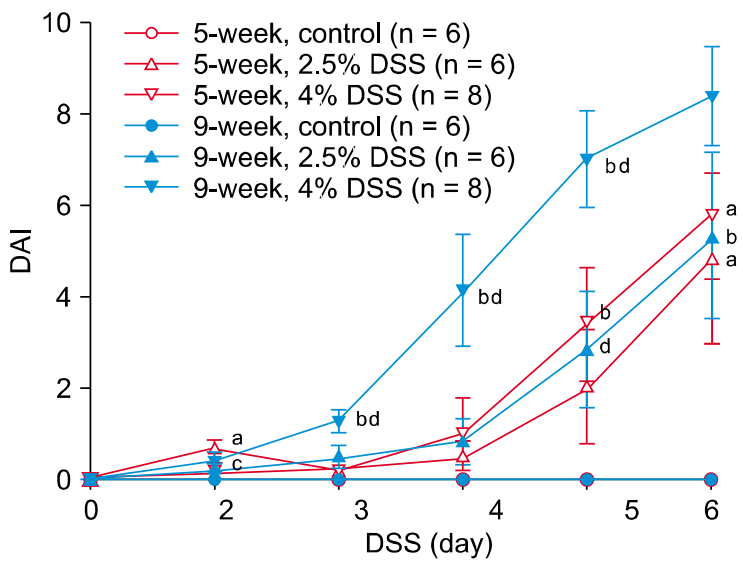

Figure 2. (A) The change of body weight was represented by ratio from administration day of study. The change in body weight show statistical difference from five days in both five- and nine-week group. ${ }^{\mathrm{b}} P<0.05$ (5-week, control vs. $2.5 \%$ DSS), ${ }^{\mathrm{a}} P<0.05$ (5-week, control vs. $4 \%$ DSS). (B) The ratio of hematochezia in mice was represented. The highest ratio group was $4 \%$ DSS in nine-week, but there was no statistical difference compared to other DSS group. ${ }^{\mathrm{a}} P<0.05$ (control vs. 5 -week), ${ }^{\mathrm{b}} P<0.05$ (control vs. 9-week). (C) Disease activity index (DAI score) are composed of the change of body weight, diarrhea, and hematochezia. The 4\% DSS mice in nine week showed the highest DAI score, and there was statistical difference compared to $4 \%$ DSS mice in five-week. Data are presented as means \pm SEMs. ${ }^{a} P$ $<0.05$ (control vs. 5-week), ${ }^{\mathrm{b}} P<0.05$ (control vs. 9-week), ${ }^{\mathrm{c}} P<0.05$ (5-week, $2.5 \%$ vs. $4 \%$ DSS), ${ }^{\mathrm{d}} P<0.05$ (4\% DSS, 5-week vs. 9-week). $D$, day of administration; DSS, dextran sodium sulfate. 
mean weight of first day (D0). The weight loss was definitely observed from Day 5 in DSS group in both 2.5\% and 4\% DSS group (Fig. 2A). Hematochezia was more frequent in $4 \%$ DSS group than $2.5 \%$ DSS group (Fig. 2B). At sacrifice day, the ratio of hematochezia of $4 \%$ DSS mice in nine-week was significantly higher than that of $2.5 \%$ DSS mice in five-week (87.5\% vs. $62.5 \%$ ), but it did not reach statistical significance $(P=0.569)$.

\section{Disease activity index score}

To evaluate the severity of colitis, DAI score were monitored daily. The DAI score was determined based on the methods of Friedman et al. ${ }^{10}$ The DAI score was calculated as the sum of the weight loss score, the diarrheal score and the hematochezia score (Table 1) and the result of daily DAI score was represented at Table 2. The DAI score of both control group in five- and nine-week was 0 in all experiment day (Table 2). In 5-week 2.5\% DSS group, DAI score began to increase from 2 day of administration and that of nine-week 4\% DSS mice markedly increased from 4 day of administration (Table 2). DAI score of both $2.5 \%$ and $4 \%$ DSS group was statistically higher than that of each control group $(P<0.05)$ (Fig. 2C). In terms of age at 4\% DSS group DAI score at nine-week showed higher score in comparison of that at five-week from DSS 3 day to DSS 6 day ( $P=0.012-0.047$ ) (Fig. 2C). However, at five-week mice only one day (DSS 2 day) of $4 \%$ DSS group showed statistical significance in comparison to $2.5 \%$ DSS group $(P=0.044)$.

Table 1. Scoring system of disease activity index ${ }^{a}$

\begin{tabular}{cccc}
\hline Score & Weight loss $(\%)$ & Stool consistency & Hematochezia $^{\mathrm{b}}$ \\
\hline 0 & None & Normal & Absence \\
1 & $0-10$ & & \\
2 & $11-15$ & Loose stool & \\
3 & $16-20$ & & \\
4 & $>20$ & Diarrhea & Presence \\
\hline
\end{tabular}

${ }^{a} \mathrm{DAI}=($ score of weight loss $)+($ score of stool consistency $)+($ score

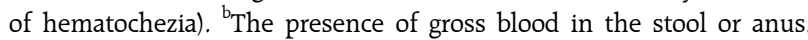
'The formation of a stool that readily becomes paste on anus of mice.

Table 2. Disease activity index score

\begin{tabular}{|c|c|c|c|c|c|c|c|c|}
\hline Week & Group & D0 & D1 & D2 & D3 & D4 & D5 & D6 \\
\hline \multirow[t]{3}{*}{ 5-week } & Control & 0 & 0 & 0 & 0 & 0 & 0 & 0 \\
\hline & $2.5 \%$ DSS & 0 & 0 & $0.67 \pm 0.21^{\mathrm{a}}$ & $0.17 \pm 0.17$ & $0.5 \pm 0.34$ & $2 \pm 1.26$ & $4.83 \pm 1.87$ \\
\hline & $4 \%$ DSS & 0 & 0 & $0.13 \pm 0.13^{\mathrm{a}}$ & $0.25 \pm 0.25^{\mathrm{b}}$ & $1 \pm 0.76^{b}$ & $3.38 \pm 1.24^{\mathrm{b}}$ & $5.75 \pm 1.41^{b}$ \\
\hline \multirow[t]{3}{*}{ 9-week } & Control & 0 & 0 & 0 & 0 & 0 & 0 & 0 \\
\hline & $2.5 \%$ DSS & 0 & 0 & $0.17 \pm 0.17$ & $0.5 \pm 0.22$ & $0.83 \pm 0.48$ & $2.83 \pm 1.28$ & $5.33 \pm 1.8$ \\
\hline & $4 \%$ DSS & 0 & 0 & $0.38 \pm 0.18$ & $1.25 \pm 0.25^{\mathrm{b}}$ & $4.13 \pm 1.23^{b}$ & $7 \pm 1.04^{b}$ & $8.38 \pm 1.07^{b}$ \\
\hline
\end{tabular}

Values are presented as mean only or mean \pm SEM. D, day of administration; DSS, dextran sodium sulfate. ${ }^{a} P<0.05(5$-week, $2.5 \%$ vs. $4 \%$ DSS). ${ }^{b} P<0.05$ (4\% DSS, 5-week vs. 9-week).

A

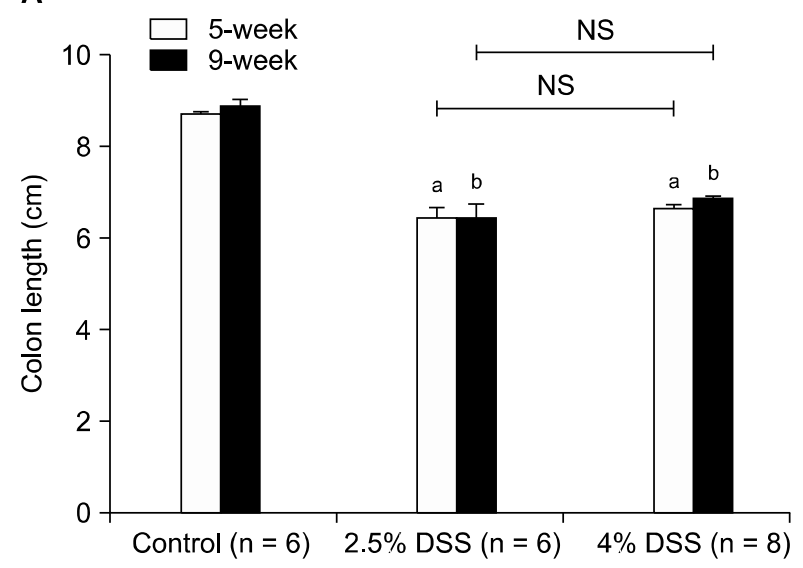

B

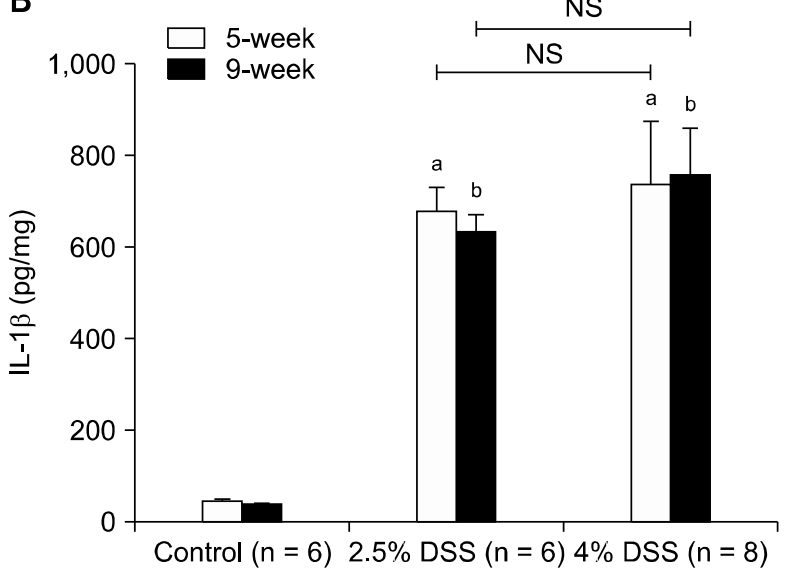

Figure 3. (A) The colon length of control and dextran sodium sulfate (DSS) group after sacrifice. The colon length of all DSS group was significantly short than that of control group. But there was no statistical difference between $2.5 \%$ and $4 \%$ DSS group. ${ }^{\text {a }} P<0.05$ (control vs. 5-week), ${ }^{\mathrm{b}} P<0.05$ (control vs. 9-week). (B) The level of interleukin 1 beta (IL-1 $\beta$ ) in the colonic mucosa was measured by reverse transcription-PCR. The level of IL-1 of all DSS group was significantly higher than that of control group. But there was no statistical difference between $2.5 \%$ and $4 \%$ DSS group. Data are presented as mean \pm SEM. ${ }^{a} P<0.05$ (control vs. 5-week), ${ }^{\text {b }} P<0.05$ (control vs. 9-week). 


\section{Colon lengths}

The colon lengths of all mice were measured at sacrifice day 7 . The mean colon length of DSS group was significantly shorter than that of control group in both five- and nine-week mice (Fig. $3 \mathrm{~A})$. The colon lengths of $2.5 \%$ DSS in five-week was slightly shorter than that of nine-week mice, but the results were not statistically significant ( $P=0.871$ ) (Fig. 3A). In addition, there was no statistical significance between $2.5 \%$ and $4 \%$ DSS mice in nine-week (6.4 \pm 0.35 vs. $6.83 \pm 0.82, P=0.362)$.

\section{Production of interleukin 1 beta}

At five-week DSS group, the level of IL-1 $\beta$ (pg/mg) expression of colonic mucosa was much higher than that of control group (control, $43.8 \pm 6.8 ; 2.5 \%$ DSS, $675.3 \pm 52.9 ; 4 \%$ DSS, $732.0 \pm$ 138.3). Similarly, in nine-week DSS mice group, the level of IL-1 $\beta$ (pg/mg) in the colonic mucosa was also much higher than control group (control, 35.2 \pm 7.3; 2.5\% DSS, 625.9 \pm 43.0; 4\% DSS, 753.4 \pm 101.1 ) (Fig. 3B). There was a great difference of IL-1 $\beta$ level between control and DSS group $(P<0.05)$. However, there was no significant difference of IL- $1 \beta$ level between $2.5 \%$ and $4 \%$ DSS group or between five- and nine-week (Fig. 3B).

\section{Histopathological analysis}

No damage was observed histologically in the colon mucosa of control group mice in 5- and 9-week in the AC and DC (Fig. 4A 4D). The crypts were straight and the base of the tubular glands reached the muscularis mucosa. The epithelial cell layer on the surface of the mucosa was intact (Fig. 4A 4D). In contrast, DSS groups showed destruction of crypts and infiltration of inflammatory cell, which were more frequently observed in DC (Fig. 4F, 4H, 4J, and 4L) than in AC (Fig. 4E, 4G, 4I, and 4K). The microscopic findings of AC showed partial crypt distortion and infiltration of inflammatory cells. Nearly complete crypt destruction and marked infiltration of inflammatory cells were observed in DC (Fig. 4F, 4H, 4J, and 4L). However, ulcer of colonic mucosa was rarely observed in colonic mucosal of DSS group.

When the microscopic histological damage score was calculated based on microscopic colonic epithelial damage and infiltration of inflammatory cell the score of DC was higher than that of AC (Fig. 5). The microscopic damage score had statistical significance in DC of DSS mice than AC in 4\% DSS group of five-week (AC
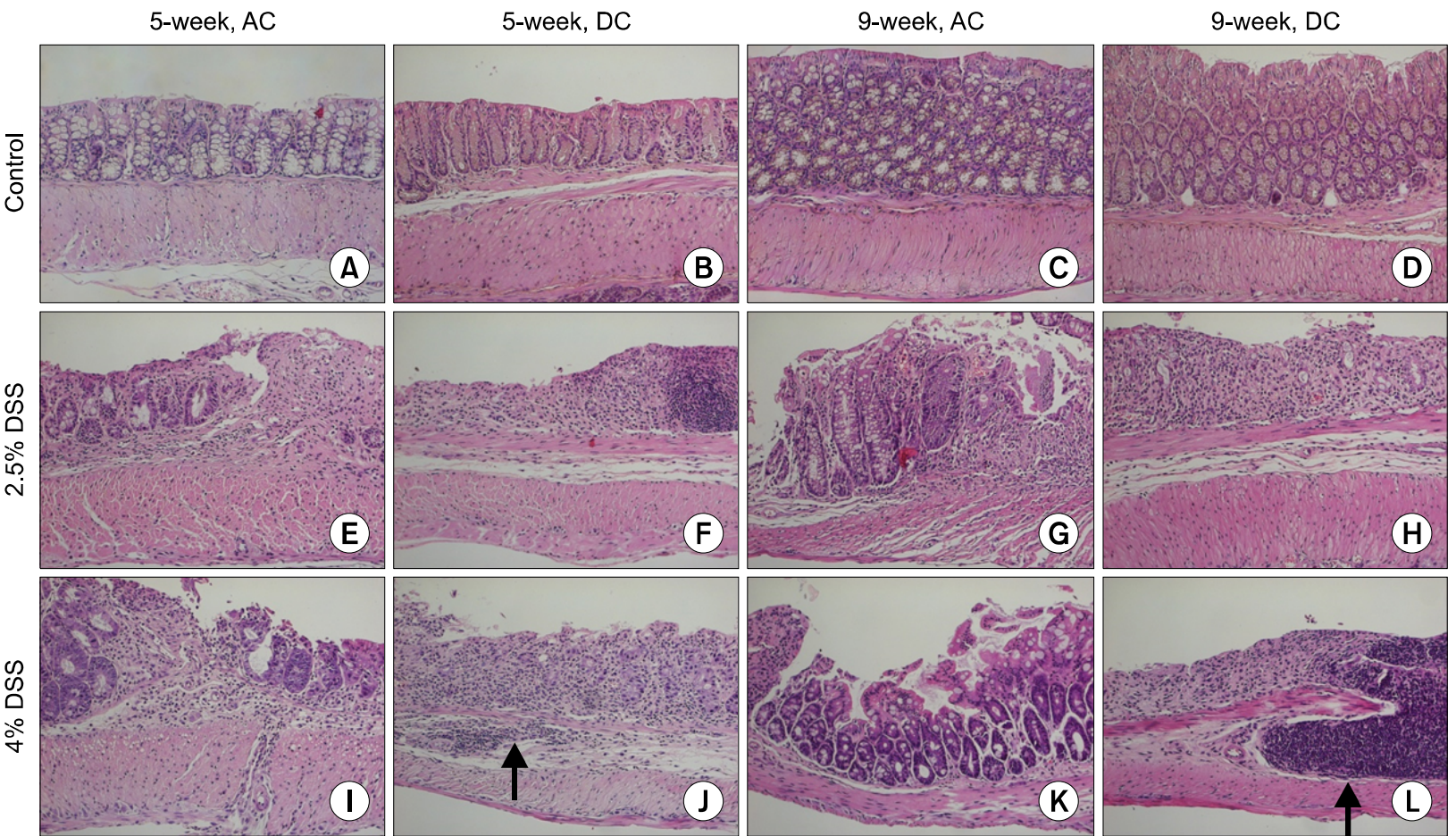

Figure 4. Histopathologic findings of the colonic mucosa (H\&E, $\times 200)$. Normal colonic mucosa of control group in five-week AC (A), DC (B); control group in nine-week AC (C), DC (D); infiltration of inflammatory cell and crypts loss (50\%) at colonic mucosa of $2.5 \%$ DSS in five-week AC (E), marked infiltration of inflammatory cell and nearly total loss of crypts at colonic mucosa of $2.5 \%$ DSS in five-week DC (F); colonic mucosa of $2.5 \%$ DSS in nine-week AC (G), DC (H); colonic mucosa of $2.5 \%$ DSS in nine-week AC (I), nearly total crypts loss and infiltration of severe inflammatory cell of colonic mucosa and submucosa (arrow) (J); 4\% DSS in nine-week AC (K), erosion and infiltration of lymphocyte (arrow) at DC (L). AC, ascending colon; DC, descending colon; DSS, dextran sodium sulfate. 


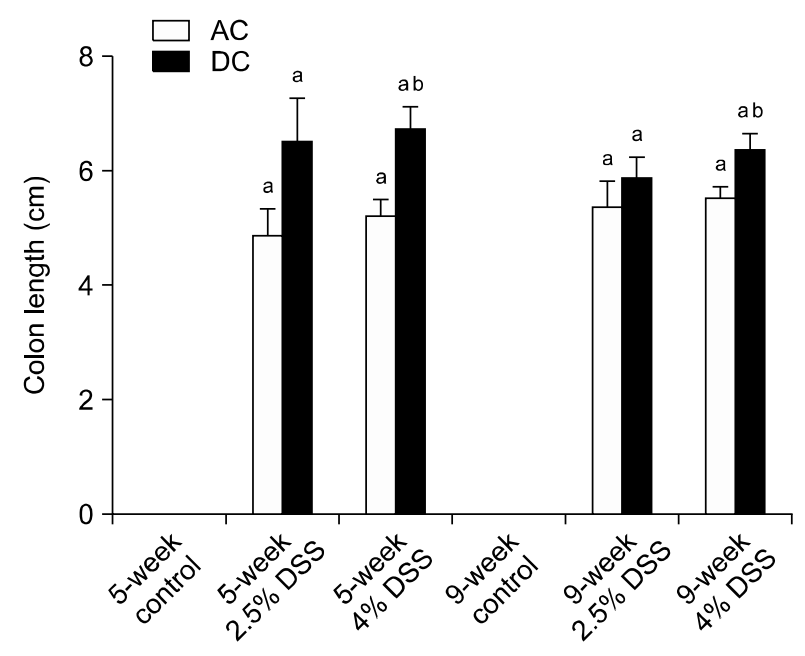

Figure 5. Microscopic damage score. The damage score of DC are more higher that that of AC. The comparison of DC and AC of $4 \%$ DSS group in five- and nine-week showed statistical significance $(P$ $<0.05$ ). Data are presented as mean \pm SEM. ${ }^{a} P<0.05$ (control vs DSS), ${ }^{\mathrm{b}} P<0.05$ (AC vs. DC). AC, ascending colon; DC, descending colon; DSS, dextran sodium sulfate.

of $4 \% \mathrm{DSS}, 5.17 \pm 0.31$; DC of $4 \% \mathrm{DSS}, 6.67 \pm 0.42 ; P=0.026$ ) (Fig. 5). The microscopic damage score had statistical significance in DC of DSS mice than AC in 4\% DSS group of nine-week (AC of $4 \%$ DSS, $5.50 \pm 0.49$; DC of $4 \%$ DSS, $6.63 \pm 0.33 ; P=0.043$ ) (Fig. 5). However, there was no statistical significance of the microscopic damage score between DSS concentration or age of mice.

\section{DISCUSSION}

IBD is comprised of two major disorders, UC and CD of which epidemiology has been published in various countries. The recent systemic research shows that the highest incidence area of IBD are Europe and North America and the low incidence area are Asia and the Middle East. ${ }^{12}$ In South Korea, a population-based study showed that the prevalence rates of UC and CD has rapidly increased for 20-year. ${ }^{13}$ IBD decreases quality of life due to low remission rate and frequent recurrences. In addition, it also increases the risk of colorectal cancer, which is related to the anatomic extent and duration of the disease. ${ }^{14}$ Actually, the colorectal cancer in the patients of IBD showed poor prognosis than sporadic colorectal cancer. ${ }^{15}$ Thus, well treatment of IBD improves the quality of life and prevents the development of colorectal cancer.

Treatment options for IBD, such as 5-aminosalicylic acid, corticosteroids, antibiotics, immunosuppressive agents (e.g., tacrolimus) and biologics (e.g., infliximab) are selectively used depending on the severity and location of the disease and the tolerability of side effects. ${ }^{16,17}$ Many different kinds of animal models have been established to study IBD. ${ }^{3}$ Especially, selection of effective animal model is very important to develop new IBD medicine. In the present study, we used DSS because of its simplicity, low coast and short time to make acute IBD mice model. Administration of DSS in drinking water induces acute mucosal injury characterized by diarrhea, body weight loss, hematochezia, and shortening of colon length. This symptom would be induced by direct hyperosmotic injury to epithelial cells. ${ }^{18}$ In addition, weight loss of DSS mice is the evidence of inflammation compared to the control group. ${ }^{9}$ However, this inflammatory reaction could be different depending on concentration of DSS. As the usual concentration of DSS was found to be $2.5 \%$ or $4 \%$ we decided to compare two concentrations in the present experiment. In the next mice age for DSS model should be decided before effective experiment. For example the inflammatory reaction of small bowel to NSAID was very different depending on age in the rat. ${ }^{19}$ Actually, several research articles used mice with variable age for DSS-induced acute colitis model. ${ }^{9,20,21}$ In the present study, all DSS mice had sign of acute inflammatory colitis than control group, and the colon length was significantly changed in all DSS mice in the present study. However, it was very difficult to choose adequate conditions such as age ( 5 or 9 weeks) and concentration of DSS (2.5\% or $4 \%$ ) by one measurement tool because there was no significant difference in the body weight, diarrhea, hematochezia, colon length, microscopic pathology, and the level of IL- $1 \beta$ of mice colon. Instead, when DAI score was applied which is calculated by combination of weight loss, diarrhea and hematochezia, 4\% DSS group in nine-week was found to be adequate IBD mice model than any other combinations. Therefore, nine-week 4\% DSS mice was an adequate DSS-induced colitis as an IBD model when assessed by DAI. In addition, this results confirms that DAI score is very important and effective tool in the selection of conditions for the IBD mice model.

IL-1 $\beta$ is an inflammatory cytokine produced predominantly by activated macrophages and monocytes, ${ }^{22}$ and its increased production has been revealed at both the mRNA and protein levels in human IBD. ${ }^{23}$ The enhanced production of IL-1 $\beta$ has been reported in DSS-induced colitis models. ${ }^{24,25}$ In addition, it is regarded as a critical cytokine in the pathogenesis of the murine colitis. ${ }^{26}$ In the present study, IL-1 $\beta$ level in the DSS group was much higher than control group, but IL-1 $\beta$ did not show a statistical difference among different ages of mice or concentration of DSS. Thus our results suggest that DAI is more useful 
indicator than IL-1 $\beta$ level in the selection of effective DSS-induced mice IBD model.

As the grading of colonic inflammation by histology is rather difficult and expensive it is very important to select the proper site of inflammation There has been a report that histopathological damage was more commonly observed in DC than in $\mathrm{AC}^{27}$ However, other study described that inflammation score of the middle colon was most higher than in the proximal or in the distal colon of mice. ${ }^{28}$ However, as the length of mice colon is rather short we chose AC and DC instead of middle colon for histologic examination. In the present study, there was no definite ulcer in any conditions. In terms of site the microscopic damage score was higher in DC than in AC. However, when the severity of histologic inflammation was compared, there was no statistical significance among groups classified by concentration of DSS or age of mice. These results suggest that distal part of colonic mucosa would be more proper to evaluate epithelial damage and infiltration of inflammatory cell than proximal part of colonic mucosa. In addition, DAI score is more effective method than histology in the measurement of inflammation or drug response in the DSS-induced mice model.

In spite of several strong points our results also have some limitations. First, the sample size was rather small. Second, two conditions for the age of mice or concentration of DSS was performed based on literature. Actually, this study was a preparatory experiment before the measurement of drug response in the DSS-induced IBD model. Thus this study was focused to find out the simple conditions for DSS-induced mice model and to set up efficient measurement tool for the inflammation of DSS mice model.

In conclusion, our study demonstrated that $2.5 \%$ and $4 \%$ DSS induced significant inflammatory reaction in both 5- and 9-week mice. Among them, nine-week 4\% DSS mice was found to be an optimal IBD model as assessed by DAI score though histological change, level of IL-1 $\beta$ didn't show significant changes depending on DSS concentrations or mice age. DAI score was useful indicator and descending colon was more appropriate site for histological evaluation of colitis than AC. Therefore, this study helps to establish the simple and clinically effective DSS-induced colitis in mice as a model of IBD.

\section{ACKNOWLEDGMENTS}

This work was supported by grant no. 06-2015-149 from the Seoul National University Bundang Hospital Research fund.

\section{CONFLICTS OF INTEREST}

No potential conflicts of interest were disclosed.

\section{REFERENCES}

1. Ng SC, Bernstein CN, Vatn MH, Lakatos PL, Loftus EV Jr, Tysk C, et al; Epidemiology and Natural History Task Force of the International Organization of Inflammatory Bowel Disease (IOIBD). Geographical variability and environmental risk factors in inflammatory bowel disease. Gut 2013;62:630-49.

2. Sands BE. From symptom to diagnosis: clinical distinctions among various forms of intestinal inflammation. Gastroenterology 2004:126:1518-32.

3. Mizoguchi A. Animal models of inflammatory bowel disease. Prog Mol Biol Transl Sci 2012;105:263-320.

4. Yan Y, Kolachala V, Dalmasso G, Nguyen H, Laroui H, Sitaraman SV, et al. Temporal and spatial analysis of clinical and molecular parameters in dextran sodium sulfate induced colitis. PLoS One 2009; 4:e6073.

5. Ohkusa T. Production of experimental ulcerative colitis in hamsters by dextran sulfate sodium and changes in intestinal microflora. Nihon Shokakibyo Gakkai Zasshi 1985;82:1327-36.

6. Wirtz S, Neufert C, Weigmann B, Neurath MF. Chemically induced mouse models of intestinal inflammation. Nat Protoc 2007;2:541-6

7. Okayasu I, Hatakeyama S, Yamada M, Ohkusa T, Inagaki Y, Nakaya R. A novel method in the induction of reliable experimental acute and chronic ulcerative colitis in mice. Gastroenterology 1990;98:694-702.

8. Chassaing B, Aitken JD, Malleshappa M, Vijay-Kumar M. Dextran sulfate sodium (DSS)-induced colitis in mice. Curr Protoc Immunol 2014;104:Unit 15.25.

9. Perše M, Cerar A. Dextran sodium sulphate colitis mouse model: traps and tricks. J Biomed Biotechnol 2012;2012:718617.

10. Friedman DJ, Künzli BM, A-Rahim YI, Sevigny J, Berberat PO, Enjyoji $\mathrm{K}$, et al. From the Cover: CD39 deletion exacerbates experimental murine colitis and human polymorphisms increase susceptibility to inflammatory bowel disease. Proc Natl Acad Sci U S A 2009;106:16788-93.

11. Katakura K, Lee J, Rachmilewitz D, Li G, Eckmann L, Raz E. Toll-like receptor 9-induced type I IFN protects mice from experimental colitis. J Clin Invest 2005;115:695-702.

12. Molodecky NA, Soon IS, Rabi DM, Ghali WA, Ferris M, Chernoff $\mathrm{G}$, et al. Increasing incidence and prevalence of the inflammatory bowel diseases with time, based on systematic review. Gastroenterology 2012;142:46-54.

13. Yang SK, Yun S, Kim JH, Park JY, Kim HY, Kim YH, et al. Epidemiology of inflammatory bowel disease in the Songpa-Kangdong district, Seoul, Korea, 1986-2005: a KASID study. Inflamm Bowel Dis 2008;14:542-9.

14. Triantafillidis JK, Nasioulas G, Kosmidis PA. Colorectal cancer and inflammatory bowel disease: epidemiology, risk factors, mechanisms of carcinogenesis and prevention strategies. Anticancer Res 2009:29:2727-37.

15. Richards ME, Rickert RR, Nance FC. Crohn's disease-associated carcinoma. A poorly recognized complication of inflammatory 
bowel disease. Ann Surg 1989;209:764-73.

16. Triantafillidis JK, Merikas E, Georgopoulos F. Current and emerging drugs for the treatment of inflammatory bowel disease. Drug Des Devel Ther 2011:5:185-210.

17. Mowat C, Cole A, Windsor A, Ahmad T, Arnott I, Driscoll R, et al; IBD Section of the British Society of Gastroenterology. Guidelines for the management of inflammatory bowel disease in adults. Gut 2011;60:571-607.

18. Schwartz L, Abolhassani M, Pooya M, Steyaert JM, Wertz X, Israël $\mathrm{M}$, et al. Hyperosmotic stress contributes to mouse colonic inflammation through the methylation of protein phosphatase 2A. Am J Physiol Gastrointest Liver Physiol 2008;295:G934-41.

19. Lee BH, Kim N, Nam RH, Lee JY, Lee HS, Lee CH, et al. Difficult establishment of a chronic nonsteroidal anti-inflammatory drugs induced gastric inflammation rat model due to gastric adaptation and small bowel damage. Korean J Gastroenterol 2014;63:341-7.

20. Kim JJ, Shajib MS, Manocha MM, Khan WI. Investigating intestinal inflammation in DSS-induced model of IBD. J Vis Exp 2012;(60). pii: 3678.

21. Laroui H, Ingersoll SA, Liu HC, Baker MT, Ayyadurai S, Charania MA, et al. Dextran sodium sulfate (DSS) induces colitis in mice by forming nano-lipocomplexes with medium-chain-length fatty acids in the colon. PLoS One 2012;7:e32084.

22. March CJ, Mosley B, Larsen A, Cerretti DP, Braedt G, Price V, et al.
Cloning, sequence and expression of two distinct human interleukin-1 complementary DNAs. Nature 1985:315:641-7.

23. Cappello M, Keshav S, Prince C, Jewell DP, Gordon S. Detection of mRNAs for macrophage products in inflammatory bowel disease by in situ hybridisation. Gut 1992;33:1214-9.

24. Villegas I, Alarcón de la Lastra C, Orjales A, La Casa C. A new flavonoid derivative, dosmalfate, attenuates the development of dextran sulphate sodium-induced colitis in mice. Int Immunopharmacol 2003;3:1731-41.

25. Tsune I, Ikejima K, Hirose M, Yoshikawa M, Enomoto N, Takei Y, et al. Dietary glycine prevents chemical-induced experimental colitis in the rat. Gastroenterology 2003:125:775-85.

26. Sävendahl L, Underwood LE, Haldeman KM, Ulshen MH, Lund PK. Fasting prevents experimental murine colitis produced by dextran sulfate sodium and decreases interleukin-1 beta and insulin-like growth factor I messenger ribonucleic acid. Endocrinology 1997;138:734-40.

27. Dieleman LA, Palmen MJ, Akol H, Bloemena E, Peña AS, Meuwissen SG, et al. Chronic experimental colitis induced by dextran sulphate sodium (DSS) is characterized by Th1 and Th2 cytokines. Clin Exp Immunol 1998;114:385-91.

28. Cooper HS, Murthy SN, Shah RS, Sedergran DJ. Clinicopathologic study of dextran sulfate sodium experimental murine colitis. Lab Invest 1993;69:238-49. 